\title{
The establishment of cow's milk protein allergy in infants is related with a deficit of regulatory T cells (Treg) and vitamin D
}

\author{
Laura Perezabad', Jacobo López-Abente², Elena Alonso-Lebrero ${ }^{3}$, Elena Seoane ${ }^{2,4}$, Marjorie Pion² and Rafael Correa-Rocha²
}

BACKGROUND: Cow's milk protein allergy (CMPA) is the most common food allergy in infants. However, little is known about which specific immune mechanisms are related with the CMPA onset. The objective was to investigate which immune alterations constitute differential factors between allergy and tolerance, and hence could be implicated in the CMPA establishment in infants.

METHODS: An extensive analysis of immune subsets, including Treg and cytokine-secreting cells was performed in blood samples from 28 infants younger than 9 mo obtained 1-4 d after the first adverse reaction to milk.

RESULTS: Less than $4 \mathrm{~d}$ after first allergic reaction, infants who developed CMPA had decreased Treg counts and increased frequency of IL4-secreting CD4 T cells compared to controls. The deficit of Tregs was correlated with decreased serum levels of vitamin D. Values of Tregs, IL4-secreting cells and vitamin D were good predictors of CMPA diagnosis. Basal vitamin D levels in CMPA infants also predicted those CMPA patients developing spontaneous tolerance in the first year.

CONCLUSION: Establishment of CMPA in infants was related with lower Treg and vitamin D levels. These immune alterations would be crucial factors behind the CMPA establishment and they could constitute a therapeutic target for treatment of CMPA.

$\mathbf{F}$ ood allergy is the most frequent reason for anaphylactic reactions in children. CMPA may occur in different clinical phenotypes according to the implication of IgE and cellular immunity in the allergic reaction (1): (i) classical IgE-mediated allergy, (ii) combined IgE and cell-mediated, and (iii) non-IgE-mediated allergy where cellular immunity is responsible of the allergy. Between the food allergies, IgE mediated Cow's milk protein allergy (CMPA) is the most common in infants during the first year of life, with a prevalence between 2 and 5\% $(2,3)$. Values of total IgE or allergen-specific serum IgE can be used for identifying an IgE-mediated allergic reaction to milk, but positive results alone are not necessarily predictive for food challenge outcome (4). An oral challenge test is considered the gold standard in confirming an adverse reaction to CMP (5).

$\mathrm{CD} 4+\mathrm{Th} 2$ cells are considered the major mediators for promoting the immune cascade responsible of the allergic symptoms. However, little is known about which immune mechanism or alterations are responsible for triggering this inflammatory cascade that produce the onset of an allergy. Considering that Th2 cells are present in the exposed tissues of any subject, why just some children develop food allergy and others do not?. Which immune alteration constitutes the differential factor between developing allergy or tolerance remains unclear, and this question needs to be elucidated to improve the prevention, the diagnosis and the treatment of these food allergies.

Regulatory T cells is a subset of CD4+ T cells with suppressive capacity (6), which have proven to prevent inadequate immune responses such us allergic processes (7). Several authors have proved in animal models both, that proallergic inflammatory status is related with an impairment or deregulation of the Treg subset, and that Treg cells are essential to promote tolerance to food allergens. Noval-Rivas et al. demonstrated that group 2 innate lymphoid cell (ILC2s) promote allergic inflammation by blocking Treg function (8). Mast cells and IgE antibodies, which trigger Th2-cell-mediated hypersensitivity reactions are also able of impairing Treg cell induction (9). In other hand, oral tolerance for food antigens can be induced in mice by transfer of Tregs (10), and oral immunotherapy (11) or low doses of IL-2 (12) are able to induce Treg-mediated control of experimental food allergies. The key role of Treg in allergy have been also confirmed in patients, showing that food-allergic infants have impaired Treg responses $(13,14)$, and that Treg cells from food-allergic children lose their suppressive function and acquire a Th2 phenotype (15). Regarding the role of Treg in promoting tolerance, Shreffler et al. (16) demonstrated that a higher frequency of allergen-specific Tregs correlates with a phenotype of mild clinical disease and favorable prognosis in

\footnotetext{
The first two authors contributed equally to this work.

'Department of Bioactivity and Food Analysis of the CIAL-CSIC, Madrid, Spain; ${ }^{2}$ Laboratory of Immune-regulation, Hospital General Universitario Gregorio Marañón and Instituto de Investigación Sanitaria Gregorio Marañón (IiSGM), Madrid, Spain; ${ }^{3}$ Pediatric-Allergy Division, Hospital General Universitario Gregorio Marañón, Madrid, Spain; ${ }^{4}$ Immunology and Allergy Pediatric Division, Hospital General Universitario Gregorio Marañón, Madrid, Spain. Correspondence: Rafael Correa-Rocha (rafael.correa@iisgm.com) 
milk allergic children. In addition, we have recently described that desensitization in egg-allergic children was mediated by a recovery in the quantity of regulatory $\mathrm{T}$ cells (Treg), and the subsequent switch in CD4 $\mathrm{T}$ cells from an activated to a hypoproliferative phenotype $(14,17)$.

However, most of these studies were performed in patients with an already established allergy. In this context, is difficult to know if the Treg impairment is a consequence of the allergic inflammatory environment in the patient as suggested by Noval-Rivas et al. (7), or whether a pre-existing Treg deficit is one of the factors responsible of the establishment of allergic phenotype. Therefore, we performed an exhaustive immune analysis including Treg values to identify the immune factors implicated in the establishment of CMPA in infants after the first adverse reaction to cow milk.

\section{METHODS}

\section{Patients and Sample Processing}

Infants younger than 9 mo, with symptoms compatible with Cow's Milk Protein Allergy (CMPA), such us vomiting or skin eruptions after cow milk ingestion were enrolled in the study at Pediatric Allergy Division or Urgencies of the Hospital. The study was conducted after the approval of the ethics committee and according to the principles expressed in the Declaration of Helsinki. Peripheral blood samples $(<3 \mathrm{ml})$ were taken after the informed consent from legal guardians before the oral challenge with cow's milk. Patients with a diagnosis of non-IgE-mediated allergy, cow's milk intolerance, allergic proctocolitis, enterocolitis, and so on were excluded from the study.

The confirmation of CMPA diagnosis was performed by an oral challenge test (unless contraindicated by severe clinical profile), and considering the total and specific IgE values, physical examination, and family history. Then, the children enrolled in the study were distributed in two groups: (i) infants with a confirmed diagnosis of CMPA (CMPA group) by immediate symptoms after oral challenge $(n=15)$; (ii) age-matched non-allergic controls (control group) $(n=$ 13) with a negative result for the oral challenge test, and subsequent ingestion of milk at home without symptoms. Blood samples were obtained 1-4 d after the first adverse reaction and just before the oral challenge test and the diagnosis of the infants. Egg allergy, which is also frequent at this age was discarded in all enrolled patients.

Patients included in the CMPA group were clinically followed along a year to determine which patients become tolerant to cow milk. A patient was considered tolerant by the absence of allergic reaction after a controlled exposition to milk, and the tolerance was confirmed if the patients were able to include in their diet a normal quantity of milk without adverse reactions.

\section{Analysis of Immune Subsets and Cytokine-Secreting Cells}

Blood samples were processed immediately after the extraction. We determined by flow cytometry in a Gallios Cytometer (Beckman Coulter, France) the frequency and absolute counts of CD4+ T cells and $\mathrm{CD} 8+\mathrm{T}$ cells, including markers for the following subsets: naive (CD45RA+CD27+), activated (HLA-DR+), central memory (CD45RA-CD27+), effector memory (CD45RA-CD27-), as previously described (18). To obtain precise physiological values of Tregs, we analyzed "untouched" total blood stained with surface markers and without employing any washing step that could modify the physiological values of cells. In this way, percentage and absolute counts of Treg in peripheral blood were quantified measuring CD3+CD4+CD25+CD127low cells. Because Foxp3 is considered the marker that better defines the Treg phenotype, percentage of Foxp3+ Treg cells (CD3+CD4+CD25+Foxp3+) were also analyzed in isolated peripheral blood mononuclear cells (PBMCs) by intracellular staining with the Anti-Human Foxp3 Staining Set (eBiosciences, San Diego, CA) according to the manufacturer's instructions. Frequency of $\mathrm{CD} 3+\mathrm{CD} 4+\mathrm{CD} 25+\mathrm{CD} 127$ low Tregs in total blood and CD3+CD4+CD25+Foxp3+ cells in isolated PBMCs were comparable in each sample in agreement with Liu et al. (19).
TemRA (CD45RA+CD27-), and RTE (CD45RA+CD27+CD31+) subsets of CD25+Foxp3+ Treg cells were also analyzed to determine the proportion of these phenotypes in the Foxp3+ Treg subset. Percentage and absolute counts of B cells (CD19+CD3-) including naive $(\mathrm{CD} 27-\operatorname{IgD}+)$, memory non-switch $(\mathrm{CD} 27+\operatorname{IgD}+)$, memory switch (CD27+IgD-) and Breg (CD24highCD27high) phenotypes; and Basophils (CD45lowCD123+IgE+) including activated basophils $(\mathrm{CD} 63+)$ were also measured in total blood. Absolute numbers of immune subtypes were determined using Flow-Count Fluorospheres (Beckman-Coulter).

Frequency of cytokine-secreting CD4+ T cells was analyzed in isolated PBMCs which were activated for $5 \mathrm{~h}$ with PMA $(50 \mathrm{ng} / \mathrm{ml})$ and Ionomycin $(1 \mu \mathrm{g} / \mathrm{ml})$, including the addition of a golgi-stopper. Intracellular staining of IL-4, IFN- $\gamma$, and IL-17 was done following the instructions of the Cytofix/Cytoperm Kit (Beckton Dickinson), and samples were acquired by flow cytometry. Frequency of cytokinesecreting cells was calculated in gated CD4+ T cells.

\section{Analysis of Vitamins and Immunoglobulins}

Total IgE and specific IgE to whole milk, BLG ( $\beta$-lactoglobulin), ALA $(\alpha$-lactalbumin) and casein IgE were measured using commercial kits (CAP-Phadia, Sweden). Serum values of IgE were expressed as kU/l.

Vitamin D was quantified as 25-hydroxyvitamin D in serum samples by chemiluminiscence. Quantification was performed employing the LIASON 25-OH-Vitamin D Total Assay in a LIASON XL analyser (DiaSorin, Stillwater, MN). Values were expressed as $\mathrm{ng} / \mathrm{ml}$.

\section{Statistical Analysis}

Statistical analysis was performed using SPSS software (IBM, New York). Nonparametric Mann-Whitney test was used for comparison between groups. Data are showed as mean \pm SEM. Correlation between variables was established by Pearson correlation (PC) test. Capacity of discrimination between groups for the different variables was analyzed by receiver operating characteristic (ROC) curves. $P$ value $<0.05$ by two-sided test was considered significant.

\section{RESULTS}

\section{Diagnosis of CMPA in Enrolled Infants}

Infants younger than 9 mo old were enrolled in the study after informed consent, and they were distributed in a control $(n=$ 13) or CMPA group $(n=15)$ attending to the results of the oral challenge test as described in methods. There were no significant differences in sex and age between groups, being the mean age in control and CMPA groups 6.18 and 6.43 mo respectively (Supplementary Table S1 online). As expected, total and specific IgE values in plasma were increased in the CMPA group but not in the control group (Supplemental Table S1 online).

\section{Extensive Analysis of Immune Subsets and Cytokines in Infants With CMPA}

Percentage and absolute counts (cells per $\mu$ l of total blood) of a wide range of immune subsets were compared between children with CMPA and controls (Table 1). Interestingly, there were very few differences between the values observed in CMPA and control children, and percentages and absolute counts for CD4 and CD8 T cells, B cells, and Basophils were comparable between both groups. We only found significant differences for naive CD8 T-cell counts, which were lower in CMPA children than in controls (Table 1).

Helper CD4+ $\mathrm{T}$ cells are essential for the initiation and maintenance of IgE-mediated allergic responses (20), and the release of Th2 cytokines plays a pathogenic role in the inflammatory changes seen in milk-induced gastrointestinal disorders (21). We stimulated in vitro PBMCs from infants and we 


\section{Articles | Garciaet al.}

Table 1. Immune parameters in children with CMPA and controls

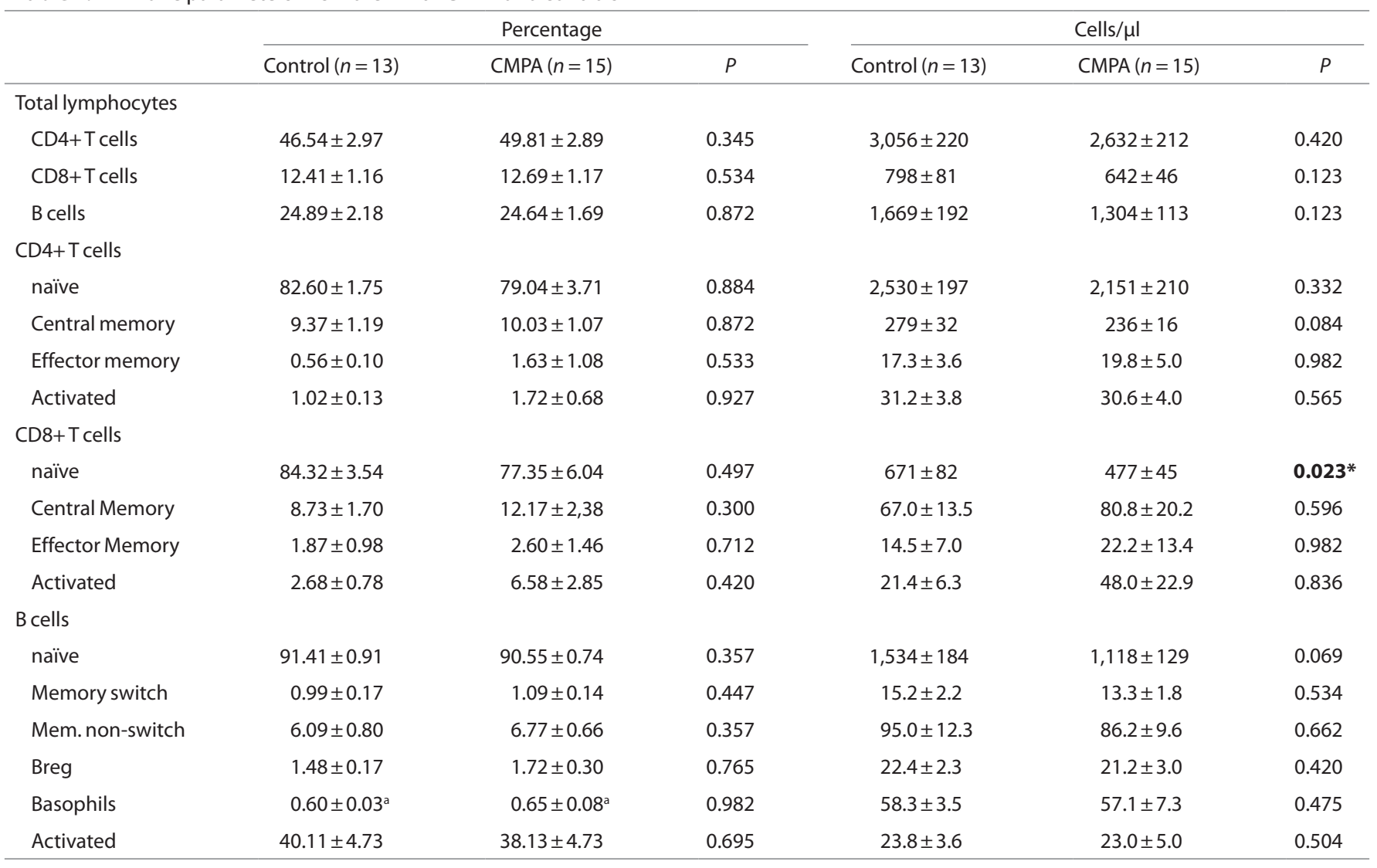

Variables with statistical significance are shown in bold.

Percentages and absolute counts (cells per $\mu$ l of total blood) for immune subsets are showed. Values are given as mean \pm SEM.

aPercentage of basophils in total leucocytes (CD45+).

${ }^{*} P<0.05$ in nonparametric Mann-Whitney test comparing control and CMPA values. Percentage for each subset is calculated regarding the total of the corresponding population (total lymphocytes; CD4+T cells; CD8+T cells; B cells or Basophils).

CMPA, cow's milk protein allergy.

analyzed the percentage of cytokine-secreting $\mathrm{CD} 4+\mathrm{T}$ cells. We did not find differences in the frequency of IFN- $\gamma$ secreting CD $4+\mathrm{T}$ cells $(\mathrm{Th} 1)($ mean $\pm \mathrm{SEM}$ CMPA $=3.51 \pm 2.09$; control $=1.82 \pm 0.34 ; P=0.742)$. There were no differences in the frequency of IL17-secreting CD4+ T cells (Th17) $(\mathrm{CMPA}=$ $0.14 \pm 0.03$; control $=0.10 \pm 0.02 ; P=0.727)$. However, the frequency of IL4-secreting CD4+ T cells (Th2) was significantly higher in CMPA children (Figure 1) $(\mathrm{CMPA}=0.69 \pm 0.13$; control $=0.43 \pm 0.06 ; P=0.037$ ).

Between all the variables studied here, the higher frequency of IL4-secreting CD4+ T cells (IL4-TCD4) seems to be the only differential immune factor in CMPA children that could be associated with the appearance of symptoms and the development of this allergy.

\section{Values of Regulatory T Cells (Treg) and Immune Homeostasis}

One of the main roles of Treg subset is to react against an inadequate proliferation of immune cells, producing regulatory cytokines or developing other regulatory mechanism that prevent an excessive expansion of effector/activated cells. This regulatory function is mediated by the production of IL-2 by activated nonregulatory $\mathrm{T}$ cells, which promotes

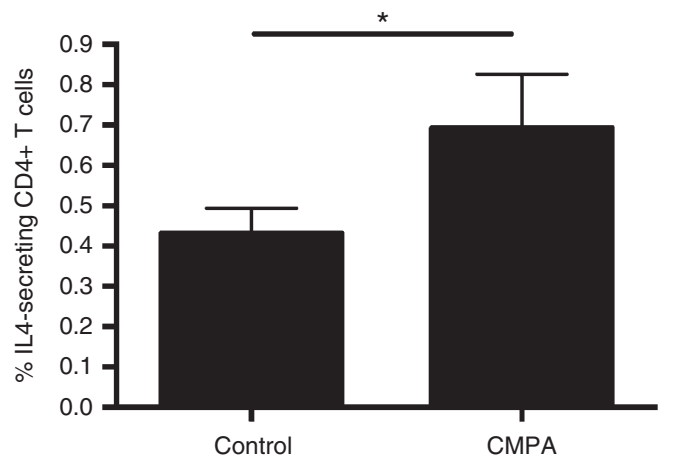

Figure 1. Percentage of IL4-secreting CD4+ T cells. Percentage of IL4secreting CD4+ T cells measured by intracellular staining in activated PBMCs. Median with interquartile range in both control and cow's milk protein allergy (CMPA) groups are represented. ${ }^{*} P<0.05$ in nonparametric Mann-Whitney test comparing control and CMPA values.

the maintenance, expansion, and activation of Tregs, which in turn limits the expansion of nonregulatory $\mathrm{T}$ cells (6). Therefore, increased frequency of (effector) IL-4 secreting $\mathrm{CD} 4+\mathrm{T}$ cells should promote the expansion/activation of Treg cells in CMPA patients. The percentage of Treg cells into the CD4+ T-cell population, which is a relative measure that can 
be influenced by the expansion or depletion of other CD4+ T subsets, was comparable between both groups (Figure 2a; $P=$ 0.433). However, when absolute counts of Treg cells (cells per $\mu$ of total blood) were measured, we found significantly lower Treg numbers in the CMPA group than in controls (Figure 2b; $P=0.040)$.

We analyzed the phenotype and the differentiation stage of these Treg cells, according to the markers defined for CD4+ $\mathrm{T}$ cells (22) and Treg cells (23). We observed that in comparison to controls, the absolute counts of all the Treg subsets were lower in the CMPA group (Supplementary Table S2 online), and this deficiency was significant for the Central Memory (Figure 2c; $P=0.040$ ) and the TemRA (Figure 2d; $P=0.022$ ) Treg subsets. Additionally, we observed only in the CMPA group a negative correlation between the frequency of IL4TCD4 cells and naive Foxp $3+\mathrm{CD} 25+$ Treg cells $(\mathrm{PC}=-0.783$; $P=0.003)$; and a positive correlation with the frequency of activated Treg $(\mathrm{PC}=0.881 ; P=0.000)$. In other words, a highfrequency IL4-TCD4 cell is associated with a decrease in the proportion of naive Treg and an increase in the proportion of activated Treg, which could reflect an active differentiation of Treg cells from a naive to an activated phenotype in response to the increased frequency of IL4-TCD4 cells.

In addition, the ratio of the Treg/IL4-TCD4 cells was lower in the CMPA group than controls, reflecting a Treg imbalance in CMPA patients. The differences in the Treg/IL4-TCD4 ratio were significant in both cases, when percentage $(P=0.036)$ or absolute counts $(P=0.027$, Figure 2e) of Treg and IL4-TCD4 cells were used to calculate the ratio between these subsets. The Treg/IL4-TCD4 imbalance and the deficit in the number of circulating Treg cells in CMPA infants could result in an inadequate control of effector $\mathrm{T}$ cells, and could explain the increased frequency of IL4-TCD4 cells. In fact, in the CMPA group but not in controls, there was a negative correlation between Treg absolute counts and IL4-TCD4 cells. The lower was the quantity of circulating Treg the higher was the frequency of IL4-TCD4 cells $(\mathrm{PC}=-0.614 ; P=0.019)$.

\section{Mechanisms of Treg Deficit: Thymic Function and Vitamin D Levels}

There are different mechanisms that could explain the decreased Treg numbers observed in the CMPA group. Because Treg cells is a subset of CD4+ T cells, which is also generated in the thymus, a deficiency in the thymic production of Treg cells could be related with the reduced number observed in periphery. We analyzed the expression of CD31 within the pool of Foxp3+CD25+ Treg cells, a marker which is only expressed in recent thymic emigrants (RTE) and it is considered an indirect indicator of thymic production (24). The results showed that there are not any significant differences in the frequency $(P=$ $0.703)$ and absolute counts $(P=0.560)$ of RTE Treg between the CMPA and control group (Figure 3a,b), discarding then a possible defect in the thymic production as the reason of Treg deficiency.

Other mechanism that could be implicated in a deficit of Treg cells in the context of allergy is the serum levels of 25-hydroxyvitamin D. This vitamin has demonstrated pronounced immunoregulatory properties and notable capacity to induce Treg cells in humans (25). Interestingly, vitamin D insufficiency/deficiency early in life has been identified as one of the risk factors for food allergy. We observed that values of vitamin $\mathrm{D}(\mathrm{ng} / \mathrm{ml})$ were significantly lower in the CMPA group than in control children $(\mathrm{CMPA}=35.3 \pm 3.5$; control $=$ $47.9 \pm 3.7 ; P=0.041$; Figure $3 \mathrm{c}$ ). Moreover, we observe a direct a

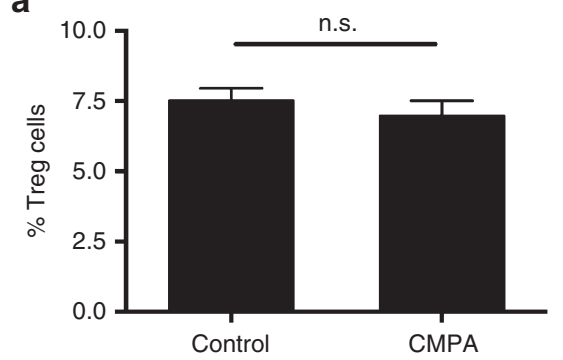

d

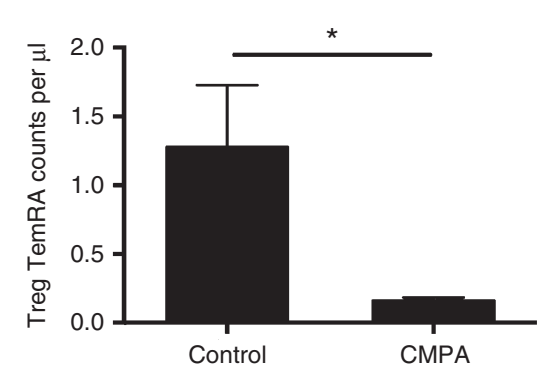

b

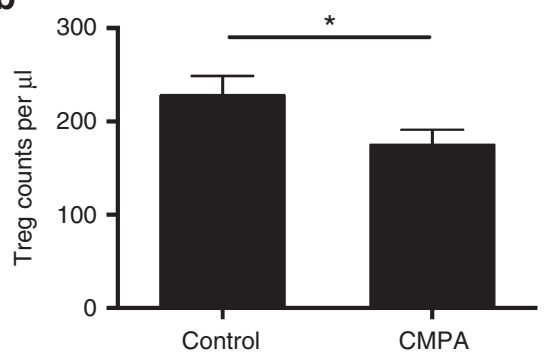

e

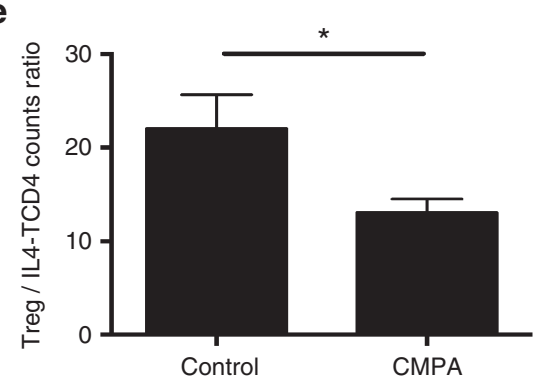

Figure 2. Values of Treg cells. Percentage (a) and absolute counts (cells per $\mu$ l of total blood) (b) of Treg cells; absolute counts of central memory (CMem) Treg cells (c) and TemRA Treg cells (d); Ratio between Treg and IL4-secreting CD4+ T cells (IL4-TCD4) absolute counts (e). Median with interquartile range in both control and cow's milk protein allergy (CMPA) groups are represented. ${ }^{*} P<0.05$, n.s.: nonsignificant differences in nonparametric Mann-Whitney test comparing control and CMPA values. 


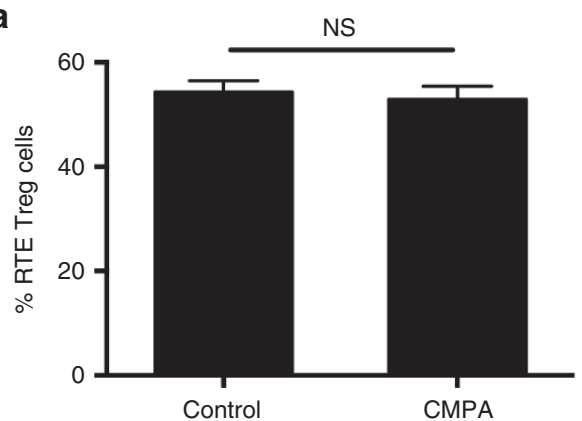

C

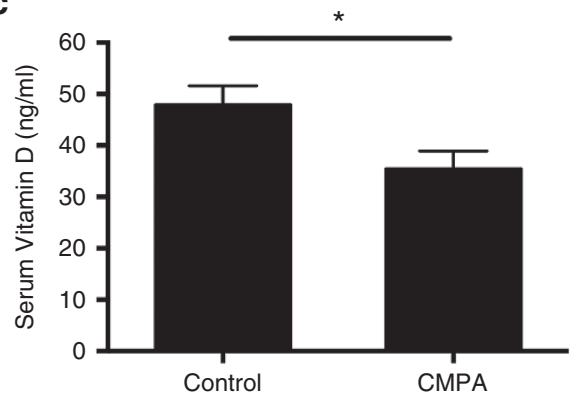

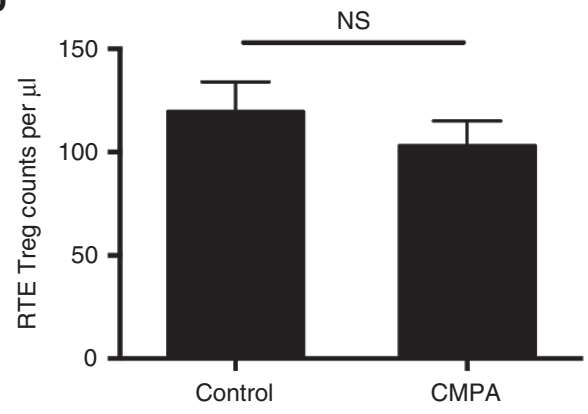

d

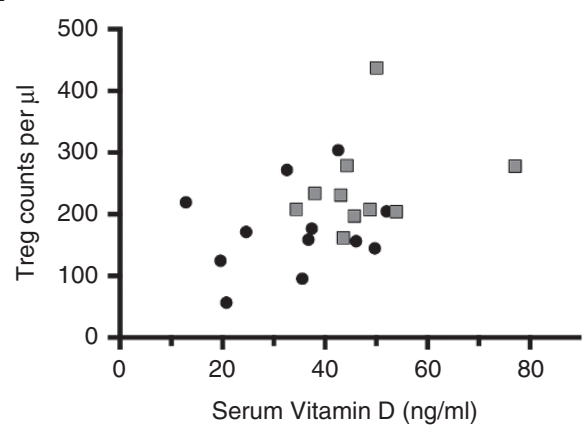

Figure 3. Values of RTE Treg cells and serum vitamin D levels. Percentage (a) and absolute counts (cells per $\mu$ l of total blood) (b) of recent thymic emigrants (RTE) Treg cells. (c) Serum concentration of vitamin D ( $\mathrm{ng} / \mathrm{ml}$ ) measured by chemiluminiscence. Median with interquartile range in both control and cow's milk protein allergy (CMPA) groups are represented. n.s.: nonsignificant differences ${ }^{*} P<0.05$ in nonparametric Mann-Whitney test comparing control and CMPA values. (d) Correlation of vitamin D levels and absolute counts of Treg cells (cells per $\mu$ l of total blood). Control: gray squares; CMPA: black dots. Pearson coefficient $=0.390 ; P=0.027$.

correlation between plasma levels of Vitamin D and absolute counts of Treg $(\mathrm{PC}=0.390 ; P=0.027)$. Serum 25 -hydroxyvitamin $\mathrm{D}$ values lower than $30 \mathrm{ng} / \mathrm{ml}$ are considered insufficient in children (26). Even if the median values of vitamin $\mathrm{D}$ in the CMPA group are higher than this cut-off, we observed that the only four children with values lower than $30 \mathrm{ng} / \mathrm{ml}$ all belonged to the CMPA group (Figure 3d). In addition, children included in the CMPA group were those with lower vitamin D values and lower Treg counts (black dots, Figure 3d), supporting the hypothesis that the deficiency in vitamin $\mathrm{D}$ could be related with the deficit of Treg cells and the subsequent increased Th2 immune responses.

\section{Treg Counts and Serum Vitamin D Levels Can Discriminate Between Healthy Controls and CMPA Children}

Blood samples to perform immune analysis were obtained from patients with a suspicion of CMPA before the oral challenge test and the definitive diagnosis of CMPA. Therefore, we analyzed whether the three variables differentially expressed in CMPA children could be good predictors of the result in the oral challenge test and the clinical diagnosis of this allergy. The ROC curve is widely utilized to evaluate the performance of diagnostic tests. The area under the ROC curve (AUC) is a widely used summary index to determine the quality to predict an event by different variables (27). ROC curves were calculated to provide information on the sensitivity and specificity of these variables to discriminate between healthy controls and CMPA children. The analysis of the data indicates that low vitamin D levels (AUC $=0.754 ; 95 \%$ confidence interval $(95 \% \mathrm{CI})=0.553-0.955 ; P=0.041)$; low absolute number of Treg (AUC $=0.728 ; 95 \% \mathrm{CI}=0.538-0.918 ; P=0.040$ ) and a high frequency of IL4-secreting CD4+ T cells $(\mathrm{AUC}=0.747$; $95 \% \mathrm{CI}=0.547-0.946 ; P=0.037)$ are good variables to discriminate between CMPA and control children (Figure 4a-c respectively). The Treg/IL4-TCD4 counts ratio was also a good predictor (AUC $=0.756 ; 95 \% \mathrm{CI}=0.536-0.946 ; P=0.027)$ to distinguish between CMPA and control children (Figure 4d).

Basal Vitamin-D Levels Were Associated With the Achievement of Spontaneous Tolerance to Cow's Milk in the First Year

Finally, we clinically followed the allergic patients included in the CMPA group to determine which patients become spontaneously tolerant to cow's milk. A patient was considered tolerant by the absence of allergic reaction after a controlled exposition to milk, and the tolerance was confirmed if the patients were able to include in their diet a normal quantity of milk without adverse reactions. Eight out of 15 patients included in the CMPA group (53.3\%) become tolerant or desensitized to cow's milk before of $1 \mathrm{y}$ after the CMPA diagnosis, and 7 out of 15 patients $(46.6 \%)$ remains allergic to milk. Interestingly, the vitamin D levels measured 1-4 d after the first adverse reaction to milk and before of CMPA diagnosis were good predictors of patients who spontaneously acquire tolerance or remain allergic. We observed in our cohort that the presence of basal levels of vitamin D lower than $40 \mathrm{ng} / \mathrm{ml}$ predicted with a sensitivity of $87.5 \%$ and a specificity of $80 \%$ those patients that remained 

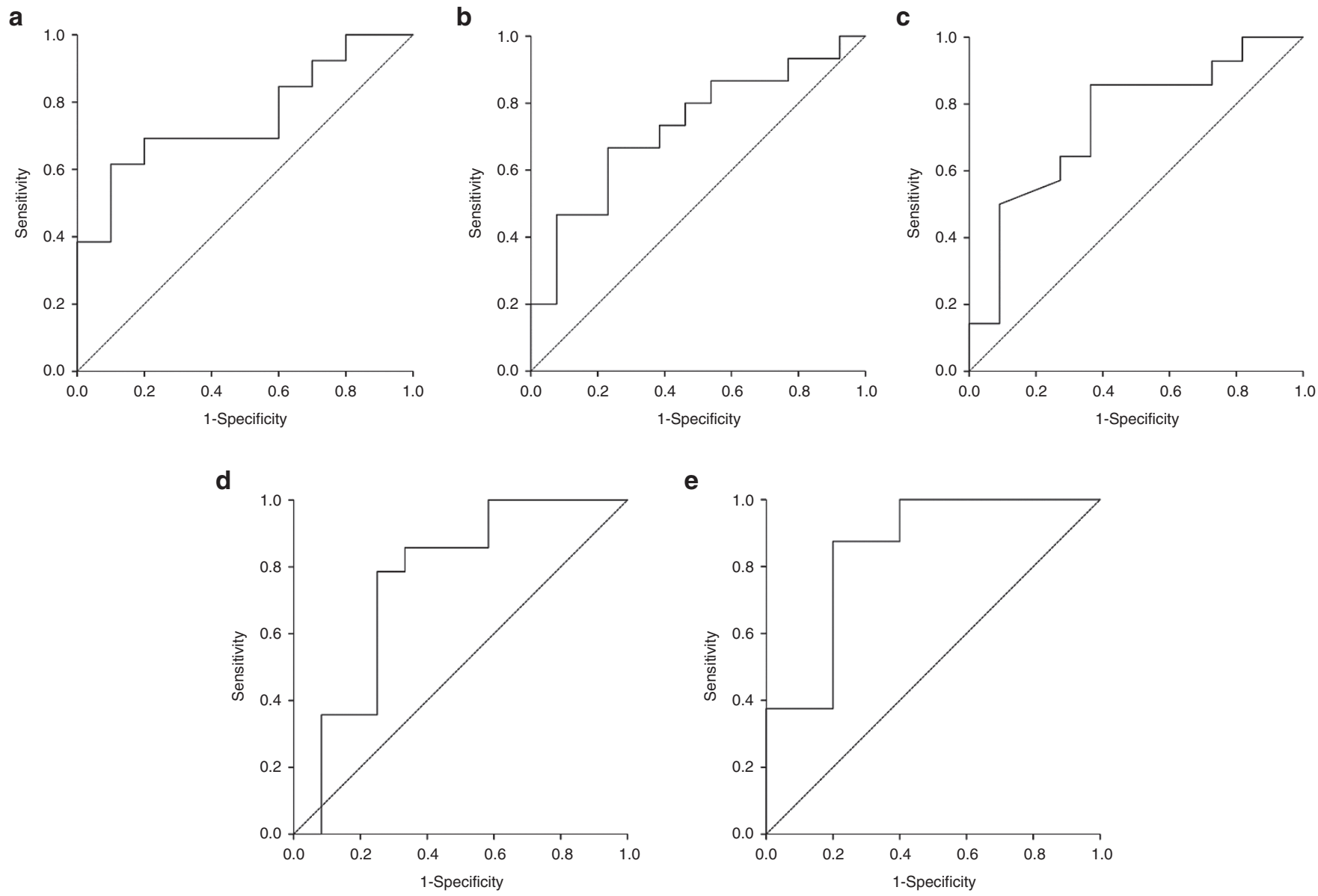

Figure 4. Receiver operating characteristic curves and predictive value of analyzed variables. Serum concentration of vitamin $D(\mu g / l)(a)$; total Treg counts (cells per $\mu$ l of total blood) (b); frequency of IL4-secreting CD4+ T cells (IL4-TCD4) (c); and the Treg/IL4-TCD4 counts ratio (d) were good discriminators between healthy and cow's milk protein allergy (CMPA) children. Basal values of vitamin D were also a good predictor of those patients that did not acquire spontaneous tolerance to cow's milk along the first year after CMPA diagnosis (e).

allergic after 1 y $(\mathrm{AUC}=0.850 ; 95 \% \mathrm{CI}=0.608-1 ; P=0.040$; Figure 4e).

These results support the hypothesis that low vitamin D levels and a deficit of circulating Treg cells could be important factors in the establishment of CMPA. However, one limitation of this study is the low $n$ value $(n=15)$. Therefore, further studies with larger cohorts of patients are required to establish whether basal Treg or vitamin D levels could constitute risk factors for CMPA onset or predictive markers of spontaneous tolerance in CMPA patients.

\section{DISCUSSION}

Here, we performed a study in infants with symptoms compatible with CMPA after cow milk ingestion, to decipher the immune alterations related with the establishment of this allergy. Samples were collected in the 1-4 d after the first adverse reaction. Therefore, we could study the immune system just after the onset of the allergic process.

Almost no difference was found in the frequency and absolute counts of the different immune subsets or in the phenotype of these populations between controls and CMPA children. In peripheral blood, the frequency of antigen-specific cells is very low (allergen-specific T cells are typically less than $0.01 \%(28)$ ), and then changes in this allergen-specific cells would not be reflected in the values of cells observed in peripheral blood. We did not analyze antigen-specific cells because the goal of our study was to identify immune markers easily measurable in peripheral blood that could be implemented in the clinical routine for the follow-up of these patients.

We observed a lower absolute number of naive CD8 T cells in CMPA children. Previous studies demonstrated a reduced percentage of CD8 T cells in children with CMPA $(29,30)$, but they do not quantify the absolute counts of these cells. Because CD8 T cells are one of the subsets responsible for the IFN-ã production, the reduced pool of naive CD8 T cells in CMPA patients could be related with the decreased production of IFN-ã observed in patients with CMPA or other atopic diseases $(29,30)$. However, the role of CD8 T cells in allergy is unclear with conflicting evidences of pathogenic or protective functions for this subset (reviewed in (31)), and further studies must be conducted to definitively determine the role of CD8 T cells in this allergy.

At the light of our results, a deficit in the number of Treg cells seems to be one of the determining factors related with 


\section{Articles | Garciaet al.}

the establishment of CMPA. Although the activation and/or differentiation of Treg cells seems to be correctly occurring in CMPA children, the frequency of IL4-TCD4 cells remains increased, probably as a consequence of the deficit in the number of circulating Treg that we observe in the CMPA group. In the case of food allergy, numerous articles in animal models and humans demonstrate how Treg cells can prevent allergic sensitization (32), induce oral tolerance $(14,33)$, and in summary how Treg cells play a crucial role in the allergic disorders (34). Some previous studies report a Treg deficiency in children with an established food allergy, and these cells have proven to be also crucial in the disappearance of the allergy (14). However, our results indicate that the Treg deficiency is already present in the first 1-4 d after the first adverse reaction. Therefore, decreased Treg values could constitute a factor that predispose for the acquisition of an atopic phenotype, and more concretely for the establishment of CMPA in infants. To definitively confirm this fact, it would be necessary to measure Treg values before of the first milk ingestion, but it is difficult to know which children will become allergic, and then hundreds of healthy children should be enrolled for this kind of study.

Regarding to the potential reasons for the Treg deficit in these infants, decreased numbers of Treg in these infants are not due to the immaturity of the immune system at this age. Previous studies demonstrate that from the birth, neonates have yet high Treg values (18). In fact, controls infants of the present study showed Treg counts around 200 cells per $\mu \mathrm{l}$, which are markedly higher than values observed in healthy children around 3 (35) or 9 y old (14). We also found that an impairment in the thymic production of Treg cells can be discarded as responsible for the decreased Treg values. RTE Treg values were not different, and naive Treg values were also comparable in both groups. That means that the defect is not at the level of Treg production or at the arrival of these naive Treg to the periphery. Between the different Treg phenotypes only the number of central memory and TemRA Treg cells were significantly reduced. These results indicate that the production, activation, and differentiation to effector cells in the Treg subset seem to develop correctly in CMPA children. In fact, we observed in the CMPA group that those children with the highest values of circulating Treg were just the children with lower frequencies of IL4-secreting CD4+ T cells, reflecting a correct suppressive function of Treg in these children. The fact that central memory and TemRA subsets of Treg (which are the most advanced steps of differentiation) are the most affected, could reflect a problem in the survival of these cells or in the mechanisms to maintain this pool after the antigenic stimulus.

Several studies demonstrate that vitamin D contribute significantly to the induction, survival, and preservation of the Treg population $(25,36,37)$. Additionally, numerous articles find a relationship between decreased values of vitamin $\mathrm{D}$, in both mother $(37,38)$ and infant $(39)$, and a higher incidence of allergy. The lower vitamin D values found in CMPA children, and the direct correlation observed between vitamin $\mathrm{D}$ and the quantity of circulating Tregs supports the hypothesis that the impaired survival of Treg cells could be influenced by the deficit of vitamin D.

We observed that IL4-secreting CD4+ T cells are the only population increased in periphery during the first phases of CMPA. Treg cells have proven to specifically prevent an excessive expansion of CD4+ T cells at the mucosa that could lead to an allergic inflammatory response (40). Numerous evidences demonstrate that Treg deficiency in the periphery is sufficient to evoke chronic T-cell-mediated autoimmunity and immunopathology (6). Therefore, the deficit of Treg found in CMPA children could be enough to favor the persistence of IL4-producing cells, which could initiate and maintain the inflammatory cascade responsible for the allergic symptoms. Moreover, a recent study demonstrates that Treg cell reprogramming toward a Th2-cell-like lineage can promote food allergy (15). The fact that the most differentiated subsets of Tregs are notably decreased could also reflect a switch of these cells to a Th2-like phenotype that will contribute to their increased frequency in CMPA children.

In summary, we hypothesized that after the introduction of cow's milk proteins in the diet, in those children with adequate immune homeostasis, Treg cells can prevent the inadequate expansion of IL4-producing CD4+ T cells. However, in children with a deficit of vitamin D probably Treg cells exerts its function initially, but without the appropriate stimulus (such as vitamin D) Tregs could have a reduced survival and become exhausted. In this scenario, the ratio of Treg/effector cells decreases and the inadequate suppression of effector cells will lead to the increased presence of IL4-secreting CD4+ T cells and the development of the allergic symptoms to these proteins. The demonstration that vitamin $\mathrm{D}$ sufficiency is an important protective factor for food allergy in the first year of life (41), supports the hypothesis that restoring the Treg survival could be a potential strategy to prevent the establishment of CMPA in infants.

Finally, the statistical analysis indicates that low vitamin D values and decreased Treg numbers, were good predictors to distinguish between controls and CMPA infants. The fact that these altered values are present few days after the first adverse reaction to the milk and prior to the definitive diagnosis of CMPA, support the utility of these values as diagnostic markers of CMPA. In addition, these parameters were good predictors of the results in oral challenge test. Therefore, Treg values and serum vitamin D levels, which are easily measurable in a blood analysis can be markers to discriminate between CMPA positive and negative, and it could replace the use of oral challenge in those patients where these tests involve a high risk. Finally, basal insufficiency of vitamin D was also a good predictor of those patients that will not achieve spontaneous tolerance in the first year, constituting also an interesting predictive marker of the clinical progression of these patients.

Further studies in larger cohorts of infants must be performed to confirm the quality of these markers, and whether Treg and vitamin D values in peripheral blood could constitute useful markers for the clinical follow-up of CMPA patients. 
SUPPLEMENTARY MATERIAL

Supplementary material is linked to the online version of the paper at http:// www.nature.com/pr

\section{ACKNOWLEDGMENTS}

The authors thank all participants in the study and their legal tutors for providing blood samples. Serum 25 -hydroxyvitamin D levels were measured by Nieves López-Lazareno (MD, PhD) at the Department of Clinical Biochemistry. The authors thank the Laura Díaz (PhD) for her technical assistance at the Flow Cytometry Unit, and Maribel Clemente Mayoral for her technical assistance and advice as responsible of the Cell Culture Facility. The authors thank all the nurses, notably Cristina Arroyo, and staff of the Pediatric-Allergy Division and Immuno-pediatrics Division of the Hospital Materno Infantil Gregorio Marañón, notably Gabriella Zambrano Ibarra (MD), for their collaboration in this project.

\section{AUTHOR CONTRIBUTIONS}

L.P. and J.L-A. were responsible of processing blood samples, the analysis of immune subsets and analysis of data. E.A-L. and E.S. were responsible of the clinical follow-up of the patients and the extraction of blood samples. They also participate in the discussion and analysis of data; M.P. contributed to the design of the project, the discussion and analysis of the data and the preparation of the manuscript; R.C-R. was responsible for the overall study, designed the project, and wrote the manuscript.

\section{STATEMENT OF FINANCIAL SUPPORT}

This work was supported by grants from Instituto de Salud Carlos III (ISCIII) co-financed by FEDER funds (PI12/00934; ICl14/00282), and by a grant from Sociedad Española de Inmunología Clínica, Alergología y Asma Pediátrica (SElCAP). R.C-R. is supported by the ISCIII through the "Miguel Servet II" program (CPII13/00033), and M.P. by the Spanish MICINN through the Ramón y Cajal Program (RYC-2009-05486). J.L-A. is supported by an IISGM pre-doctoral grant, and L.P. is in receipt of a FPU PhD Grant (AP2012-2343) from the Ministerio de Educación, Cultura y Deporte (Spain).

Disclosure: All the authors declare that they have no competing financial interests.

\section{REFERENCES}

1. Poza-Guedes P, Barrios Y, González-Pérez R, Sánchez-Machín I, Franco A, Matheu V. Role of specific IgE to $\beta$-lactoglobulin in the gastrointestinal phenotype of cow's milk allergy. Allergy Asthma Clin Immunol 2016;12:7.

2. Venter C, Pereira B, Grundy J, et al. Incidence of parentally reported and clinically diagnosed food hypersensitivity in the first year of life. J Allergy Clin Immunol 2006;117:1118-24.

3. Vandenplas Y, Abuabat A, Al-Hammadi S, et al. Middle east consensus statement on the prevention, diagnosis, and management of cow's milk protein allergy. Pediatr Gastroenterol Hepatol Nutr 2014;17:61-73.

4. Boyce JA, Assaad A, Burks AW, et al.; NIAID-Sponsored Expert Panel. Guidelines for the diagnosis and management of food allergy in the United States: summary of the NIAID-Sponsored Expert Panel Report. J Allergy Clin Immunol 2010;126:1105-18.

5. Costa AJ, Sarinho ES, Motta ME, Gomes PN, de Oliveira de Melo SM, da Silva GA. Allergy to cow's milk proteins: what contribution does hypersensitivity in skin tests have to this diagnosis? Pediatr Allergy Immunol 2011;22(1 Pt 2):e133-8.

6. Sakaguchi S, Yamaguchi T, Nomura T, Ono M. Regulatory T cells and immune tolerance. Cell 2008;133:775-87.

7. Noval Rivas M, Chatila TA. Regulatory T cells in allergic diseases. J Allergy Clin Immunol 2016;138:639-52.

8. Noval Rivas M, Burton OT, Oettgen HC, Chatila T. IL-4 production by group 2 innate lymphoid cells promotes food allergy by blocking regulatory T-cell function. J Allergy Clin Immunol 2016;138:801-811.e9.

9. Burton OT, Noval Rivas M, Zhou JS, et al. Immunoglobulin E signal inhibition during allergen ingestion leads to reversal of established food allergy and induction of regulatory T cells. Immunity 2014;41:141-51.

10. Yamashita $H$, Takahashi $K$, Tanaka $H$, Nagai $H$, Inagaki $N$. Overcoming food allergy through acquired tolerance conferred by transfer of Tregs in a murine model. Allergy 2012;67:201-9.
11. Smaldini PL, Orsini Delgado ML, Fossati CA, Docena GH. Orally-induced intestinal CD4+ CD25+ FoxP3+ Treg controlled undesired responses towards oral antigens and effectively dampened food allergic reactions. PLoS One 2015;10:e0141116.

12. Bonnet B, Vigneron J, Levacher B, et al. Low-dose IL-2 induces regulatory $\mathrm{T}$ cell-mediated control of experimental food allergy. J Immunol 2016;197:188-98.

13. Dang TD, Allen KJ, J Martino D, Koplin JJ, Licciardi PV, Tang ML. Foodallergic infants have impaired regulatory T-cell responses following in vivo allergen exposure. Pediatr Allergy Immunol 2016;27:35-43.

14. Fuentes-Aparicio V, Alonso-Lebrero E, Zapatero L, et al. Induction of Treg cells after oral immunotherapy in hen's egg-allergic children. Pediatr Allergy Immunol 2014;25:103-6.

15. Noval Rivas M, Burton OT, Wise $\mathrm{P}$, et al. Regulatory $\mathrm{T}$ cell reprogramming toward a Th2-cell-like lineage impairs oral tolerance and promotes food allergy. Immunity 2015;42:512-23.

16. Shreffler WG, Wanich N, Moloney M, Nowak-Wegrzyn A, Sampson HA. Association of allergen-specific regulatory $\mathrm{T}$ cells with the onset of clinical tolerance to milk protein. J Allergy Clin Immunol 2009;123: 43-52.e7.

17. Fuentes-Aparicio V, Alonso-Lebrero E, Zapatero L, et al. Oral immunotherapy in hen's egg-allergic children increases a hypo-proliferative subset of CD4+ T cells that could constitute a marker of tolerance achievement. Pediatr Allergy Immunol 2012;23:648-53.

18. Correa-Rocha R, Pérez A, Lorente R, et al. Preterm neonates show marked leukopenia and lymphopenia that are associated with increased regulatory T-cell values and diminished IL-7. Pediatr Res 2012;71:590-7.

19. Liu W, Putnam AL, Xu-Yu Z, et al. CD127 expression inversely correlates with FoxP3 and suppressive function of human CD4+ T reg cells. J Exp Med 2006;203:1701-11.

20. Islam SA, Luster AD. T cell homing to epithelial barriers in allergic disease. Nat Med 2012;18:705-15.

21. Beyer K, Castro R, Birnbaum A, Benkov K, Pittman N, Sampson HA. Human milk-specific mucosal lymphocytes of the gastrointestinal tract display a TH2 cytokine profile. J Allergy Clin Immunol 2002;109:707-13.

22. Mahnke YD, Brodie TM, Sallusto F, Roederer M, Lugli E. The who's who of T-cell differentiation: human memory T-cell subsets. Eur J Immunol 2013;43:2797-809.

23. Miyara M, Yoshioka Y, Kitoh A, et al. Functional delineation and differentiation dynamics of human CD4+ T cells expressing the FoxP3 transcription factor. Immunity 2009;30:899-911.

24. Kimmig S, Przybylski GK, Schmidt CA, et al. Two subsets of naive Thelper cells with distinct $\mathrm{T}$ cell receptor excision circle content in human adult peripheral blood. J Exp Med 2002;195:789-94.

25. Penna G, Roncari A, Amuchastegui S, et al. Expression of the inhibitory receptor ILT3 on dendritic cells is dispensable for induction of CD4+Foxp3+ regulatory $\mathrm{T}$ cells by 1,25-dihydroxyvitamin D3. Blood 2005;106:3490-7.

26. Muehleisen B, Gallo RL. Vitamin D in allergic disease: shedding light on a complex problem. J Allergy Clin Immunol 2013;131:324-9.

27. Yao W, Li Z, Graubard BI. Estimation of ROC curve with complex survey data. Stat Med 2015;34:1293-303.

28. Wambre E, DeLong JH, James EA, et al. Specific immunotherapy modifies allergen-specific CD4(+) T-cell responses in an epitope-dependent manner. J Allergy Clin Immunol 2014;133:872-9.e7.

29. Osterlund P, Suomalainen H. Low frequency of CD4+, but not CD8+, $\mathrm{T}$ cells expressing interferon-gamma is related to cow's milk allergy in infancy. Pediatr Allergy Immunol 2002;13:262-8.

30. Järvinen KM, Aro A, Juntunen-Backman K, Suomalainen H. Large number of CD19+/CD23+ B cells and small number of CD8+ T cells as early markers for cow's milk allergy (CMA). Pediatr Allergy Immunol 1998;9:139-42.

31. Huber M, Lohoff $M$. Change of paradigm: CD8+ T cells as important helper for CD4+ T cells during asthma and autoimmune encephalomyelitis. Allergo J Int 2015;24:8-15.

32. van den Elsen LW, Meulenbroek LA, van Esch BC, et al. CD25+ regulatory $\mathrm{T}$ cells transfer $\mathrm{n}-3$ long chain polyunsaturated fatty acids-induced tolerance in mice allergic to cow's milk protein. Allergy 2013;68:1562-70. 


\section{Articles | Garcia et al.}

33. Karlsson MR, Rugtveit J, Brandtzaeg P. Allergen-responsive CD4+CD25+ regulatory T cells in children who have outgrown cow's milk allergy. J Exp Med 2004;199:1679-88.

34. Palomares O, Yaman G, Azkur AK, Akkoc T, Akdis M, Akdis CA. Role of Treg in immune regulation of allergic diseases. Eur J Immunol 2010;40:1232-40.

35. Ferrando-Martínez S, Lorente R, Gurbindo D, et al. Low thymic output, peripheral homeostasis deregulation, and hastened regulatory $\mathrm{T}$ cells differentiation in children with 22q11.2 deletion syndrome. J Pediatr 2014;164:882-9.

36. Chambers ES, Hawrylowicz CM. The impact of vitamin D on regulatory $\mathrm{T}$ cells. Curr Allergy Asthma Rep 2011;11:29-36.

37. Vijayendra Chary A, Hemalatha R, Seshacharyulu M, Vasudeva Murali M, Jayaprakash D, Dinesh Kumar B. Vitamin D deficiency in pregnant women impairs regulatory $\mathrm{T}$ cell function. J Steroid Biochem Mol Biol 2015;147:48-55.

38. Chiu CY, Huang SY, Peng YC, et al. Maternal vitamin D levels are inversely related to allergic sensitization and atopic diseases in early childhood. Pediatr Allergy Immunol 2015;26:337-43.

39. Jones AP, D’Vaz N, Meldrum S, Palmer DJ, Zhang G, Prescott SL. 25-hydroxyvitamin D3 status is associated with developing adaptive and innate immune responses in the first 6 months of life. Clin Exp Allergy 2015;45:220-31.

40. Curotto de Lafaille MA, Kutchukhidze N, Shen S, Ding Y, Yee H, Lafaille JJ. Adaptive Foxp3+ regulatory T cell-dependent and -independent control of allergic inflammation. Immunity 2008;29:114-26.

41. Allen KJ, Koplin JJ, Ponsonby AL, et al. Vitamin D insufficiency is associated with challenge-proven food allergy in infants. J Allergy Clin Immunol 2013;131:1109-16, 1116.e1-6. 